\title{
Experiments on Chaotic Vibrations of a Thin Circular Plate with Outer Clamped Edge and a Circular Center Hole*
}

\author{
Ken-ichi NAGAI**, Kenji OKADA**, Shinichi MARUYAMA** and \\ Takao YAMAGUCHI** \\ ** Depertment of Mechanical System Engineering, Graduate School of Engineeering, Gunma University \\ 1-5-1, Tenjin-cho, Kiryu, Gunma 376-8515, Japan \\ E-mail: kennagai@gunma-u.ac.jp
}

\begin{abstract}
This paper presents experimental results on chaotic vibrations of a thin circular plate with a circular center hole. The plate has an asymmetric curved configuration due to an initial imperfection, an in-plane compressive stress and lateral deformation by the gravity. First, linear natural frequencies and natural modes of vibration are measured. The second and the third modes of vibration have one nodal diameter with different natural frequencies. These nodal diameters are perpendicular to each other. The linear natural frequencies of the lowest and the second modes satisfy the condition of oneto-two internal resonance, closely. Characteristics of restoring force of the plate show the type of a softening-and-hardening spring. Next, the plate is excited with periodic acceleration, laterally. Sweeping the excitation frequency, nonlinear responses of the plate are measured. In a restricted frequency range of the principal resonance of the lowest mode, non-periodic responses with amplitude modulation are generated. The time histories of the response are measured for a long-time interval at four positions, simultaneously. The time histories are inspected by the Fourier spectrum, the Poincaré projection, the maximum Lyapunov exponents and the principal component analysis. The non-periodic response is found to be the chaotic response generated from the oneto-two internal resonance coupled with the lowest and the second modes. The results of the principal component analysis with the time histories of the multiple positions on the plate show that the lowest, second and third modes predominantly contribute to the chaotic response. Applying the calculation with short-time intervals cut out from the time histories, it is found that the nodal patterns of the second and the third modes of vibration are fluctuated along the circumferential direction. Furthermore, the exchange of the contribution ratio is shown between the lowest and the second modes of vibration.
\end{abstract}

Key words : Nonlinear Vibration, Chaos and Fractal, Vibration of Continuous System, Circular Plate, Principal Component Analysis

\section{Introduction}

A circular plate with outer clamped edge and a circular center hole is used for a part of industrial products in piping such as orifice meters and mufflers of vehicles. When the fluid flows in the piping, vibrations are generated on the plate. In typical conditions, chaotic vibrations are generated and cause problems such as noises and breaking of the plate. The chaotic vibrations are generated under two conditions as follows. First, the temperature of the fluid is higher than that out of piping. Secondly, the thickness of the plate is sufficiently thin. The difference between temperatures in and out of the piping induces in-plane compressive stress in the plate. The in-plane compressive stress induces in-plane displacement. If the thickness of the plate is sufficiently thin, lateral deflection is coupled with in-plane displacement, in- 
tensely. Consequently, the plate shows nonlinear characteristics of restoring force. When the plate is subjected to lateral periodic acceleration, nonlinear responses are generated with resonance. Furthermore, the chaotic responses with random-like response are generated by modal interactions in typical frequency ranges. For designing safe industrial products, it is important to investigate chaotic vibrations of the thin plate.

Many researches are found on nonlinear vibrations of plates with circular boundaries. Yamaki et al. studied nonlinear vibrations of a clamped circular plate with initial deflection and initial edge displacement ${ }^{(1),(2)}$. Afarat et al. analyzed natural frequencies and nonlinear vibrations of a heated annular plate ${ }^{(3),(4)}$. Traveling waves, which are generated in a plate with circular boundary, have also been studied by many resurchers. Nayfeh T. A. et al. analyzed sub-harmonic traveling waves in a geometrically nonlinear circular plate ${ }^{(5)}$. Trii T. et al. studied nonlinear oscillation of a rotating disc excited at fixed point in space ${ }^{(6)}$. However, traveling waves in the chaotic response have not studies well, as authors know.

In this experiments, the chaotic vibrations of a thin circular plate with outer clamped edge and a circular center hole are investigated. The plate has initial imperfection and is subjected to an in-plane compressive stress. First, the configuration of the plate, linear natural frequencies, natural modes of vibration and characteristics of restoring forces of the plate are measured. Next, the plate is subjected to periodic acceleration, laterally. Nonlinear responses of the plate are measured by sweeping the excitation frequency in the neighborhood of the lowest natural frequency. In a restricted frequency range, a non-periodic response is generated. The time responses of the plate are recorded for a long-time interval at the multiple positions on the plate, simultaneously. The chaotic responses are inspected by the Fourier spectra, the Poincaré projection, the maximum Lyapunov exponents and the principal component analysis. Furthermore, to inspect the traveling waves in the chaotic response, the results of the principal component analysis are compared by changing the length of time interval for the calculation.

\section{Thin circular plate with outer clamped edge and a circular center hole}

A thin circular plate with outer clamped edge and a circular center hole is shown in Fig. 1. The outer edge of the plate is clamped by rigid rings. The inner edge of the circular center hole corresponds to the free edge. The dimension of the plate is measured as the radius of the plate $a=70.0 \mathrm{~mm}$, the radius of the hole $b=16.9 \mathrm{~mm}$ and the thickness of the plate $h=0.30 \mathrm{~mm}$. The ratio of the inner radius $b$ to the outer radius $a$ is 0.24 . For the test specimen of the plate, an aluminum sheet is used. The material properties are taken as Young's modulus $E=67.7 \mathrm{GPa}$, the mass density $\rho=2.66 \times 10^{3} \mathrm{~kg} / \mathrm{m}^{3}$ and Poisson's ratio $v=0.33$. The polar coordinate system is defined by $r$-axis along radial direction, $\theta$-axis along the circumferential direction and $z$-axis vertical to the surface of the plate.

To obtain the boundary condition of the plate, the circular plate with outer clamped edge and a circular center hole is fabricated by the following procedure. First, a circular plate with a circular center hole is obtained by cutting the aluminum sheet. The radius of the circular plate is $100 \mathrm{~mm}$ and radius of a hole is $16.9 \mathrm{~mm}$. By cutting the sheet, initial imperfection is formed in the plate. Secondly, a pair of rigid annular rings is made of duralumin. The dimension of a rigid ring is inner radius $70.0 \mathrm{~mm}$ and outer radius $100 \mathrm{~mm}$ and the thickness $15 \mathrm{~mm}$. Finally, the plate is sandwiched between the rigid rings and the rigid rings are fixed by bolts. Consequently, the outer clamped edge and the inner free edge corresponding to the hole is obtained. When the plate is fixed, the temperature of the plate is kept lower than that in the experiments. By this procedure, in-plane compressive stress is generated due to the thermal elongation of the plate. To enhance measuring accuracy of the displacement, both surface of the plate are painted with acrylic resin of white color. The thickness $h^{\prime}$ of the plate including the painted layer is $h^{\prime}=0.38 \mathrm{~mm}$. As the results of the fabrication, the plate has initial deformation under the gravity. The initial deformation of the plate is influenced by the change of the temperatures of the plate and the rigid rings. The thermal condition in the experiments is explained latter in $\$ 3.4$. 


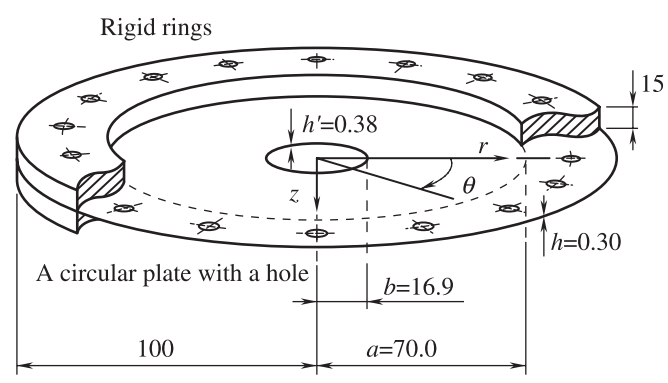

Fig. 1 A thin circular plate with outer clamped edge and a circular center hole.

\section{Vibration test apparatus and test procedures}

\subsection{Measurement of fundamental properties of the plate}

As fundamental properties of the circular plate with a hole, a configuration of plate, linear natural frequencies, modes of vibration and characteristics of restoring force of the plate are measured.

First, the configuration of the plate is measured by a laser displacement sensor. Secondly, the linear natural frequencies and modes of vibration are measured. Applying periodic acoustic pressure on the plate, resonant response with small amplitude is detected by the laser displacement sensor. The natural frequencies of the plate are inspected by the digital spectrum analyzer. The natural modes of vibration are also obtained by scanning the resonant responses over the plate. Thirdly, the relation between static deflection and a concentrated force is obtained as the characteristics of restoring force of the plate. Pressing a load cell to the plate, the concentrated force is loaded on the plate. Then, the plate deflects to an equilibrium position of elastic force between the plate and the load cell. At the equilibrium position, the static deflection and the concentrated force are recorded.

\subsection{Procedures of vibration test on chaotic response}

A schematic diagram of vibration test apparatus is shown in Fig. 2. Whole instruments of the vibration test are numbered from 1 to 17 . The plate is excited periodically with an electromagnetic exciter through the rigid ring. The rigid ring is fixed on the vibration head of the exciter. The excitation is provided by the instruments as numbered from 1 to 5 . The exciter controller 1 generates a sinusoidal periodic signal. The periodic signal is amplified with the power amplifier 2. The vibration exciter 3 drives the rigid ring with periodic acceleration through the exciter head 4 . The accelerometer pickup 5 fixed on the rigid ring detects the acceleration acting on the plate. The signal of the acceleration is fed back to the controller 1 . Then, the amplitude of periodic acceleration can be kept to a prescribed constant level during a sweep of the excitation frequency.

Dynamic deflections of the plate are measured with the instruments of the laser displacement sensor from 6 to 8 . Relative displacement of the plate to the rigid ring is detected with the laser displacement sensors 6 and 7. The sensor 6 detects the periodic displacement of the rigid ring. The sensor 7 measures the dynamic deflection of the plate and the periodic displacement of the rigid ring. The controller 8 subtracts the two signals. With this subtraction, the pure dynamic deflection of the plate can be detected. The laser sensors 7 are set on the sliding table 9 and the sensor moves on the surface of the plate.

The instruments from 10 to 17 are the measuring devices of signal processing and data analysis. Nonlinear frequency-response of the plate is obtained by sweeping the excitation frequency. The dynamic deflections of the plate detected by the laser sensors are transformed to the amplitude in a root mean square value with the digital voltmeter 10 . The excitation frequency applied on the plate is counted with the digital frequency counter 11 through the periodic signal from the exciter controller 1 . The amplitude of the dynamic response and the excitation frequency are transferred to the computer 12. Then, the nonlinear frequency- 
response curve is obtained. The digital spectrum analyzer 13 records time histories of chaotic response of the plate. The time history of the chaotic response is transformed to the Fourier spectrum. Furthermore, the time history of the chaotic time response is transmitted to the computer 12 and the maximum Lyapunov exponents $\lambda_{\max }$ are calculated with the procedure proposed by Wolf et al. ${ }^{(7)}$. The Poincaré projection of the response is obtained by the following step. Dynamic deflection of the response is transformed to velocity by the differentiation amplifier 14. The set of the deflection and the velocity is recorded sequentially once in every period of the excitation. Synchronized with the period of excitation acceleration, a pulsating signal is generated with the phase meter 15 and the delayed pulse oscillator 16. The phase meter detects the maximum amplitude of the periodic acceleration, and then the pulse oscillator generates the pulsating signal with a prescribed phase delay. The set of aforementioned deflection and velocity of the chaotic response is recorded by the analyzer 13 in each trigger by the pulsating signal. The Poincaré projection of the chaotic response is stored in the computer 12, and the projection is also displayed. The principal component analysis is adopted to the chaotic response to confirm modal contributions. The time histories of the chaotic response are simultaneously recorded for a long-time interval at four positions on the plate by multi-channel recorder 17. Multiple time histories are transferred to the computer 12 and the principal components are calculated by Karrhunen-Loève transformation ${ }^{(10)}$.

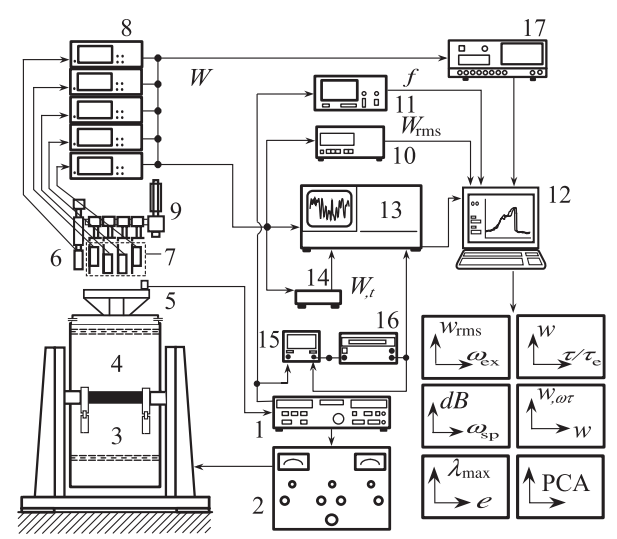

Fig. 2 Diagram of vibration test apparatus.

\subsection{Evaluation of the chaotic response}

Multiple methodologies of inspection are required to confirm the chaotic response of the plate. A Fourier spectrum of a non-periodic time history shows a broadband spectrum. However, dominant components of the spectrum are related to the periodic resonant response from which the chaotic response is generated. The Poincare projections of the chaotic response show a distributed pattern on the phase plane.

The maximum Lyapunov exponents are able to confirm the chaotic response of the plate and to find the number of vibration modes, which contribute to chaotic response. If the maximum Lyapunov exponent has a positive value as the embedding dimension is increased, the response can be confirmed as chaos. Furthermore, the half number of embedding dimensions, where the exponent converges as the dimension is increased, corresponds to the number of vibration modes contributed to the chaos. The maximum Lyapunov exponents are calculated with the procedure proposed by Wolf et al. ${ }^{(7)}$. In the experiment, $e$-dimensional pseudo-phase space is composed with time-delay coordinates from a time histories, where $e$ is the embedding dimension ${ }^{(8)}$. A component of the pseudo-phase space is a sequential time history chosen partially from the time response which has a fixed time delay from the former component. A trajectory in the pseudo-phase space is constructed from the single time response of the plate from an arbitrary start time. Two trajectories, which initially have sufficiently small distance, are selected as the fiducial trajectory and the nearby trajectory. The exponential growth rate of 
the distance between the two trajectories is calculated in each infinitesimal time interval. The exponential growth rate is averaged in the long time interval, then the maximum Lyapunov exponents $\lambda_{\max }$ can be estimated. In the experiments, time histories of the chaotic responses contain not only the chaotic response but also inevitable components such as uncertainty of measurement, external disturbances and others. In such cases, the maximum Lyapunov exponent continuously decreases as the embedding dimension is increased without converging. As a result, the estimation of convergence of the maximum Lyapunov exponents $\lambda_{\max }$ may be difficult. However, the gradient of the maximum Lyapunov exponents discontinuously changes when the embedding dimension exceeds twice of the number of vibration modes, which contributes to the chaotic response. In this experiments, convergence of the maximum Lyapunov exponents $\lambda_{\max }$ is estimated by the change in gradient of $\lambda_{\max }$ to the increase of embedding dimension.

The principal component analysis ${ }^{(10)}$ enables the estimation of contribution ratio and the modal pattern in the chaotic response. In the principal component analysis, the covariance matrix of the simultaneous time histories of the response at multiple positions on the plate is calculated. The covariance matrix is transformed to an orthogonal matrix, which results in the eigenvalue problem of the covariance matrix. The eigenvector $\phi_{i}$ represents the modal pattern and the corresponding eigenvalue denotes the contribution of the modal pattern to the chaotic response. Contribution ratio $\mu_{i}$ is the ratio of eigenvalues of the $i$ th modal pattern to the all modal patterns.

\subsection{Thermal control}

The dynamic responses are influenced by the change of the initial deformation of the plate. The initial deformation of the plate is changed by the in-plane stress. The in-plane stress is changed by the thermal elongation of the plate relative to the rigid ring. To obtain precise results of the experiments on the chaotic vibrations of the plate, the temperatures both of the plate and rigid ring should be kept constant through the experiment. The temperatures are controlled by the thermal control system, which is used in the authors' previous study ${ }^{(9)}$. The temperature $T_{r}$ of the air in the vicinity of the plate and the temperature $T_{f}$ of the rigid ring are detected with thermal sensors. Through the experiments, the temperatures $T_{r}$ and $T_{f}$ are controlled within $20.5 \pm 0.5^{\circ} \mathrm{C}$.

\subsection{Non-dimensional notation}

To discuss the results of the experiments, the following non-dimensional notations are introduced:

$$
\begin{aligned}
& \xi=r / a,\left[w_{0}, w_{s}, w\right]=\left[W_{0}, W_{s}, W\right] / h,\left[p_{s}, p_{d}\right]=\left[g, a_{d}\right] \rho a^{4} / D, \\
& q_{s}=Q_{s} a^{2} / D h,\left[\omega_{\mathrm{ex}}, \omega_{i}\right]=\left[f_{\mathrm{ex}}, f_{i}\right]\left(2 \pi / \Omega_{0}\right), \tau=\Omega_{0} t .
\end{aligned}
$$

In the foregoing, $\Omega_{0}=\left(1 / a^{2}\right) \sqrt{D /(\rho h)}$ are used, where $D=E h^{3} /\left[12\left(1-v^{2}\right)\right]$ is the bending stiffness of the plate, $E, v$ and $\rho$ are the Young's modulus, the Poisson's ratio and the mass density of the plate, respectively. In the above notations, $\xi$ is the non-dimensional coordinate. The symbols $w_{0}, w_{s}$ and $w$ indicate non-dimensional notation of the initial deformations $W_{0}$, the static deflections $W_{s}$ and the dynamic deflections $W$, respectively. The notations $p_{s}$ and $p_{d}$ are the non-dimensional gravity $g$ and the non-dimensional amplitude of the periodic acceleration $a_{d}$. The notation $q_{s}$ is the non-dimensional concentrated force. The symbols $\omega_{\mathrm{ex}}$ and $\omega_{i}$ are the non-dimensional excitation frequency and the linear natural frequencies. The symbol $\tau$ denotes the non-dimensional time.

\section{Results and discussion}

\subsection{Fundamental properties of the plate}

The plate has initial deformation due to an initial imperfection, an in-plane compressive stress and lateral deformation by the gravity. The configuration of initial deformation of the 
plate is shown Fig. 3. The temperatures $T_{r}$ and $T_{f}$ are set as $T_{r}=T_{f}=20.5{ }^{\circ} \mathrm{C}$. At the position $\xi=0.32$ and $\theta=0$, large deformation of the plate is generated $w_{0}=1.2$. The initial deformations of plate are decreased to the clamped boundary from the position $\xi=0.32$ and $\theta=0$. Thus, the plate shows an asymmetric curved configuration. The asymmetric curved configuration of the plate influences the natural frequencies and the natural modes of vibration.

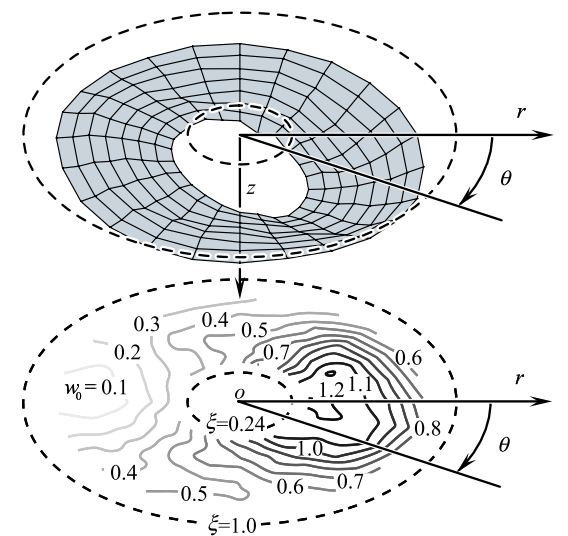

Fig. 3 The configuration of the plate under gravity.

Table 1 shows linear natural frequencies of the plate at the temperatures $T_{r}=T_{f}=20.5$ ${ }^{\circ} \mathrm{C}$. Modal patterns related to the vibration modes are also listed in the table. In the table, the broken lines on the modal patterns indicate the nodal patterns. The lowest mode of vibration forms a symmetric mode. The second and the third modes of vibration with one nodal diameter are generated in different natural frequencies by the asymmetric curved configuration. These nodal diameters are perpendicular to each other. Two modes of vibration are distinguished by natural frequencies. These modes are defined as modes $2 \mathrm{a}$ and $2 \mathrm{~b}$, respectively. In the table, the natural frequency of the mode $2 \mathrm{a}$ is nearly twice the frequency of mode 1 . Thus, the condition of one-to-two internal resonance is satisfied closely.

Figure 4 shows the characteristics of restoring force of the plate. The static deflection $w_{s}$ is shown by the concentrated force $q_{s}$ acting on $\xi=0.47$ and $\theta=0$. The static deflections are measured at four positions on the plate from the equilibrium position under the gravity. The static deflections $w_{s}$ are measured at the positions $(\xi=0.62, \theta=0),(0.62, \pi / 2),(0.62, \pi)$ and $(0.62,3 \pi / 2)$, which are marked by circle, square, triangle, and inverse triangle, respectively. The temperatures $T_{r}$ and $T_{f}$ are controlled within $T_{r}=20.3 \pm 0.1{ }^{\circ} \mathrm{C}$ and $T_{f}=20.4 \pm$ $0.2{ }^{\circ} \mathrm{C}$. Increasing the concentrated force from the equilibrium position to the negative $z$ direction, the gradient of curve of the restoring force decreases because of the influence of initial deformation of the plate. The decrease of the gradient corresponds to the characteristics of a softening spring. As the restoring force is increased further, the gradient of curve of the restoring force increases. The characteristics of hardening spring are show by the increase of the gradient of the curve. Consequently, the characteristics of restoring force of the plate show the type of a softening-and-hardening spring.

The initial deformation of the plate and natural frequencies are influenced by the change of temperatures $T_{r}$ and $T_{f}$, sensitively. For example, giving the thermal deference $1.0{ }^{\circ} \mathrm{C}$ between $T_{r}$ and $T_{f}$, by changing the thermal condition from $T_{r}=T_{f}=21.0{ }^{\circ} \mathrm{C}$ to $T_{r}=20.0$ ${ }^{\circ} \mathrm{C}$ and $T_{f}=21.0{ }^{\circ} \mathrm{C}$, the initial deformation decreases 5 percent to the thickness of the plate at the position $\xi=0.47$ and $\theta=0$. The non-dimensional natural frequency of the lowest mode increases 0.55 . These changes influence the amplitude of dynamic response.

\subsection{Frequency-response curve}

Under the excitation amplitude $p_{d}=4.4$, the frequency-response curve is obtained by sweeping the excitation frequency in the range of the frequency $\omega_{\mathrm{ex}}=6.2$ to 10.5 . The 
Table 1 Natural frequencies and natural modes of vibration.

\begin{tabular}{|c|c|c|c|c|}
\hline Mode $i$ & 1 & $2 \mathrm{a}$ & $2 \mathrm{~b}$ & 3 \\
\hline $\begin{array}{c}\text { Modal } \\
\text { pattern }\end{array}$ & 0 & $\cdots$ & $\vdots$ & $\vdots$ \\
\hline$\omega_{i}$ & 8.25 & 16.0 & 18.8 & 29.4 \\
\hline$f_{i}[\mathrm{~Hz}]$ & 124 & 242 & 284 & 444 \\
\hline
\end{tabular}

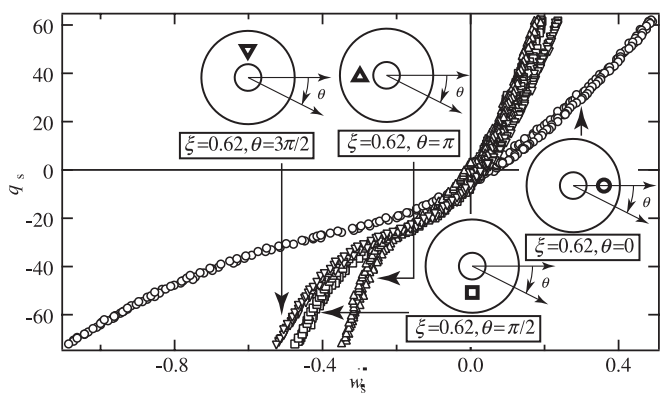

Fig. 4 Characteristics of restoring force of the plate: $\circ$ measured at $\xi=0.62, \theta=0$, $\square$ measured at $\xi=0.62, \theta=\pi / 2, \Delta$ measured at $\xi=0.62, \theta=\pi, \nabla$ measured at $\xi=0.62, \theta=3 \pi / 2$.

excitation frequency is swept very slowly to avoid transient effects on the chaotic response. The temperatures $T_{r}$ and $T_{f}$ are controlled within $T_{r}=20.5 \pm 0.5^{\circ} \mathrm{C}$ and $T_{f}=20.9 \pm 0.1^{\circ} \mathrm{C}$, respectively. The nonlinear frequency-response curve is shown in Fig. 5. The ordinate shows the non-dimensional amplitude $w_{\text {rms }}$ of the dynamic deflection $w$ with a root mean square value at the position $\xi=0.47$ and $\theta=0$. The abscissa indicates the excitation frequency $\omega_{\mathrm{ex}}$. The natural frequency of the mode 1 is also indicated by the circle on the abscissa. The frequencyresponse curve shows large amplitude resonant resonance of the mode 1 with the type of a hardening spring. When the plate is excited at the frequency $\omega_{\mathrm{ex}}=9.6$ near the half of natural frequency of the mode $2 b$, non-periodic responses are generated in a restricted range of the principal resonance of the mode 1 , which is denoted by the mark $C$. The non-periodic response shows large amplitude of vibration with amplitude modulation. The response is confirmed as the chaotic vibration with the evaluation methodology of chaotic response in the $\$ 3.3$.

Difference of amplitudes appears between the responses when the excitation frequency is swept up and down. At the same amplitude of the response $w_{\mathrm{rms}}=0.61$, the difference of the excitation frequency is 0.37 . The difference can be predominantly induced by the fluctuation of the temperatures $T_{r}$ and $T_{f}$ as explained in $\S 4.1$. As the temperatures $T_{r}$ and $T_{f}$ are changed very slowly, the effect of the fluctuation of the temperatures on the frequency-response curve is large because the time interval for recording the frequency-response curve is long. However, the effect of the fluctuation of the temperatures on the time histories is small because the time-interval for recording the time histories is shorter than the change of the temperature.

\subsection{Time history, Fourier spectrum and Poincaré projection of the chaotic response}

Under the excitation amplitude $p_{d}=4.4$, the time history, the Fourier spectrum and the Poincare projection of the chaotic response are obtained. At the excitation frequency $\omega_{\mathrm{ex}}=9.59$, the response is measured at the position $\xi=0.47$ and $\theta=0$. In Fig. 6(a), the time history of the dynamic deflection $w$ are shown with the time ratio normalized by the excitation period $\tau_{\mathrm{e}}=2 \pi / \omega_{\mathrm{ex}}$. The time history of the response has irregular amplitude modulation. Fig. 6(b) shows the Fourier spectrum of the chaotic response, where the abscissa indicates the nondimensional Fourier frequency $\omega_{\text {sp }}$, while the ordinate denotes the amplitude $A$ of the spectrum scaled by decibel. As can be seen in the Fig. 6(b), broadband spectrum is shown. Furthermore, distinguished peak spectra of the response can be detected at the excitation frequency $\omega_{\mathrm{ex}}$ 
and at twice the excitation frequency $2 \omega_{\text {ex }}$. Near the distinguished peak spectra, the natural frequencies of the modes $1,2 \mathrm{a}$ and $2 \mathrm{~b}$ exist. The Poincaré projection is shown in Fig. 6(c). The response of the dynamic deflection $w$ and the velocity $w_{, \omega \tau}$ are plotted at the phase $\psi=$ $\pi / 3$ from the maximum amplitude of the excitation acceleration. Points of the projection are distributed in wide region. Moreover, comparing the projection to that of the chaotic response generated from internal resonance, which is explained in a previous study ${ }^{(11)}$, the characteristics of the projection are similar to each other. Consequently, these characteristics of the response show that the response is a chaotic response generated from the one-to-two internal resonance. From the results of the principal component analysis as described in $\S 4.6$, it is confirmed that the chaotic response is generated from internal resonance coupled with the modes 1 and 2a.

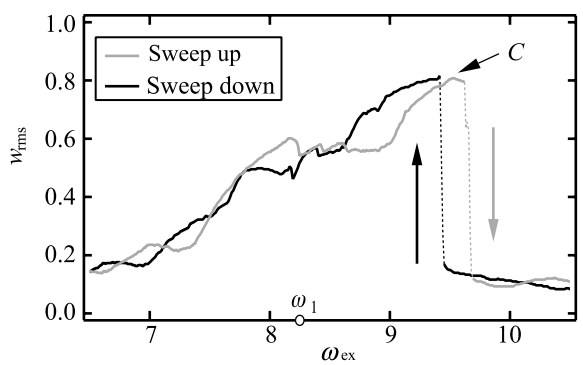

Fig. 5 Frequency response curve of the plate ( $p_{d}=4.4$, measured at $\xi=0.47, \theta=0$ ).

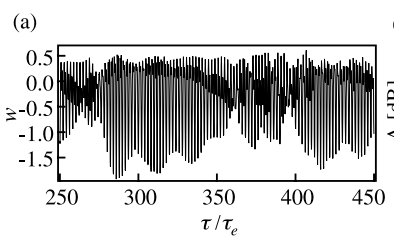

(b)

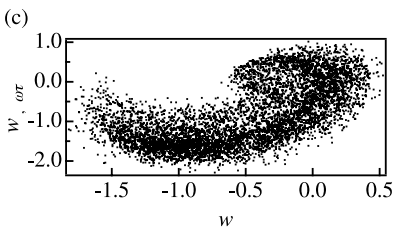

Fig. 6 Chaotic response of the plate $\left(\omega_{\mathrm{ex}}=9.59, p_{d}=4.4\right.$, measured at $\xi=0.47$, $\theta=0$ ): (a) time history; (b) Fourier spectrum; (c) Poincaré projection.

\subsection{The Fourier spectra in short-time intervals}

The chaotic response of the plate has the amplitude modulation. To investigate the frequency components related to the amplitude modulation, the Fourier spectra are calculated with the time history in short-time intervals. Under the excitation amplitude $p_{d}=4.4$, the time history of the response is measured at the position $\xi=0.47$ and $\theta=0$. The time history of the dynamic response at the excitation frequency $\omega_{\mathrm{ex}}=9.59$ and the amplitude $w_{\text {rms }}$ of the dynamic deflection with a root mean square value in short-time intervals $18.5 \tau_{\mathrm{e}}$ are shown in Fig. 7 (a) and (b), respectively. As can be seen in Fig. 7(a) and (b), it is confirmed that the amplitude of the response is changed irregularly. The Fourier spectra are calculated with the time histories in the short-time intervals. Figure 8(a) shows the Fourier spectrum inspected in the time interval $\tau / \tau_{\mathrm{e}}=2148.5$ to 2167.0 , where the amplitude of response is relatively small. The distinguished peak spectra can be detected at the excitation frequency $\omega_{\mathrm{ex}}$ and at twice the excitation frequency $2 \omega_{\mathrm{ex}}$. Furthermore, the spectra are distributed near the natural frequency of the mode 2a. Fig. 8(b) shows the Fourier spectrum inspected in the time interval $\tau / \tau_{\mathrm{e}}=2222.6$ to 2241.1 , where the amplitude of the response is large. The distinguished 
peak spectra can be also detected at the excitation frequency $\omega_{\mathrm{ex}}$ and at twice the excitation frequency $2 \omega_{\text {ex }}$. However, the distribution of the spectra near the natural frequency of the mode $2 \mathrm{a}$ disappears. Consequently, the amplitude modulation of the response is related to the mode $2 \mathrm{a}$.

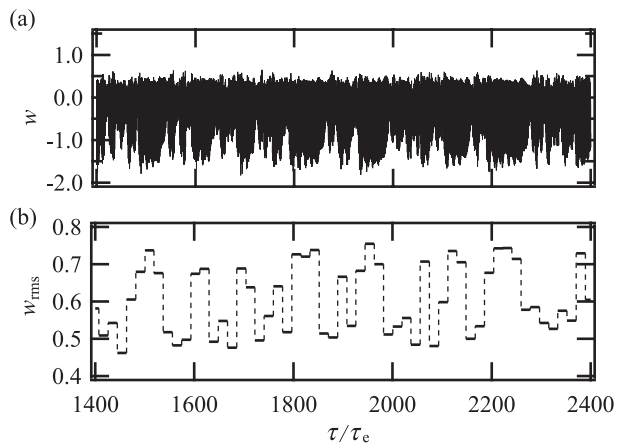

Fig. 7 The time history of the deflection and the amplitude in short time intervals $\left(\omega_{\mathrm{ex}}=9.59, p_{d}=4.4\right.$, measured at $\left.\xi=0.47, \theta=0\right)$ : (a) time history of the dynamic deflection; (b) time history of the amplitude.

(a)
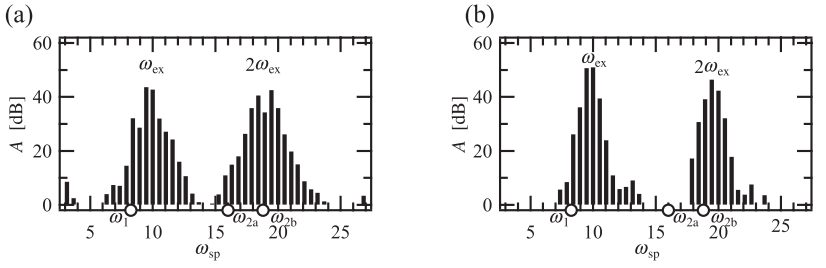

Fig. 8 The Fourier spectra in short time intervals $\left(\omega_{\mathrm{ex}}=9.59, p_{d}=4.4\right.$, measured at $\xi=0.47, \theta=0$ ): (a) $\tau / \tau_{\mathrm{e}}=2148.5$ to 2167.0 ; (b) $\tau / \tau_{\mathrm{e}}=2222.6$ to 2241.1 .

\subsection{The maximum Lyapunov exponents of the chaotic response}

To confirm the response as the chaos, the maximum Lyapunov exponents $\lambda_{\max }$ related the embedding dimension $e$ are calculated from the time history at the excitation frequency $\omega_{\mathrm{ex}}=9.59$. The position is selected at $\xi=0.47$ and $\theta=0$. Figure 9 shows the maximum Lyapunov exponent related embedding dimension. As the embedding dimension increases more than $e=7$ to 10 , the gradient of maximum Lyapunov exponents is decreased at $\lambda_{\max }=$ 0.3 to 0.4 . As the gradient of the maximum Lyapunov exponent is changed at the positive value of the exponent, the chaotic response is confirmed as the chaos. Moreover, it is found that four to five modes of vibration contribute to the chaotic response because the gradient of the maximum Lyapunov exponent is changed at the embedding dimension $e=7$ to 10 .

\subsection{Vibration modes contribute to the chaotic response}

Principal component analysis is adapted to the time histories of the chaotic response to investigate contributions of the vibration modes. The multiple time histories of the responses are measured at four positions on the plate, simultaneously. The positions are selected at $(\xi=0.47, \theta=0),(0.47, \pi / 2),(0.47, \pi)$ and $(0.47,3 \pi / 2)$. The time histories are recorded for a long-time interval $2500 \tau_{\mathrm{e}}$ at the excitation frequency $\omega_{\mathrm{ex}}=9.59$.

Figure 10 shows the contribution ratio and corresponding modal pattern of the chaotic response. The abscissa indicates the order of eigenvalues $i$, while the ordinate denotes the contribution ratio $\mu_{i}$. The largest principal component, which corresponds to the mode 1 , shows prevailing contribution ratio of 66.1 percent. The second and third larger principal components, which correspond to the modes $2 \mathrm{a}$ and $2 \mathrm{~b}$, show the contribution ratio of 24.0 and 13.9 percent, respectively. The fourth contribution, which corresponds to the mode 3 , has 


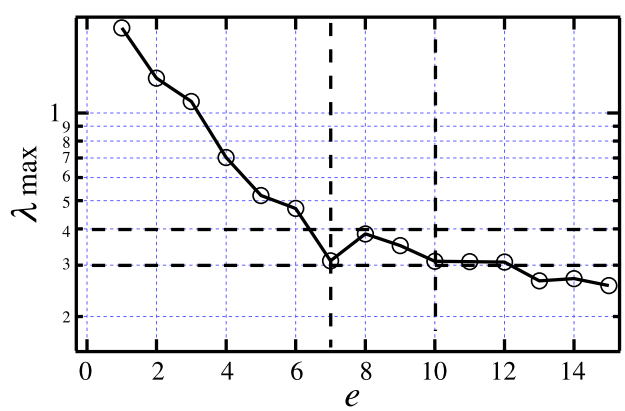

Fig. 9 The maximum Lyapunov exponents related to embedding dimension $\left(\omega_{\mathrm{ex}}=9.59, p_{d}=4.4\right.$, measured at $\left.\xi=0.47, \theta=0\right)$.

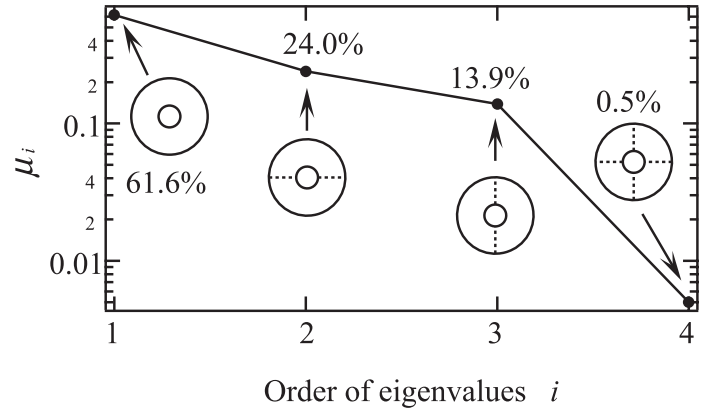

Fig. 10 Principal components obtained by Karrhunen-Loève transformation $\left(\omega_{\mathrm{ex}}=\right.$ 9.59, $\left.p_{d}=4.4\right)$.

small contribution ratio of 0.5 percent. Thus, the modes $1,2 \mathrm{a}$ and $2 \mathrm{~b}$ predominantly contribute to the chaotic response.

To investigate the motion of nodal patterns, the principal components are calculated with time histories in short-time intervals cut out from the time history of the long-time interval. The short-time intervals are taken as the $50 \tau_{\mathrm{e}}$. Figure 11 shows the time history of the amplitude $w_{\text {rms }}$ of the dynamic deflection with a root mean square value in the short-time intervals. The time histories in Fig. 11 (a) to (d) correspond to the response at the positions ( $\xi=0.47, \theta=0),(0.47, \pi / 2),(0.47, \pi)$ and $(0.47,3 \pi / 2)$, respectively. As can be seen in Fig. 11 , the amplitude of the response is changed irregularly. However, the difference between the modulations in the amplitude appears at the four positions. The modulation is large at the positions $(0.47,0)$ and $(0.47, \pi)$, while that is small at the positions $(0.47, \pi / 2)$ and $(0.47,3 \pi / 2)$. The positions $(0.47,0)$ and $(0.47, \pi)$ are located on the nodal diameter of the mode $2 \mathrm{a}$, which is related to the amplitude modulation. The large modulation at the positions $(0.47,0)$ and $(0.47, \pi)$ is relative to the change of the contribution ratio of the mode 1 in the short-time intervals, which is explained latter.

Figure 12 shows the modal pattern related to the each order of contribution ratio. In the figure, the modal patterns are denoted by circles. The lines represent the modal configurations, which obtained by the least square approximation of the modal pattern. The black thick lines and gray thin lines represent the modal configurations obtained by the principal component analysis with the long-time interval and the short-times intervals, respectively. From the result of the calculation with the long-time interval, the modal configurations in Fig. 12(a) to (d) correspond to the modes $1,2 \mathrm{a}, 2 \mathrm{~b}$ and 3 , respectively. The modal configurations in the short-time intervals show the similar forms to that in long-time interval. However, the modal configurations with different orientations from the long-time interval are found on the second and the third contribution, which correspond to the modes $2 \mathrm{a}$ and $2 \mathrm{~b}$. These configurations are distributed irregularly along the circumferential direction. The distribution of the modal configurations corresponds to the irregular fluctuation of the nodal patterns.

The time history of the contribution ratio in the short-time intervals is shown in Fig.13. 
The contribution ratio of the modal configurations, which correspond to modes $1,2 \mathrm{a}$ and $2 \mathrm{~b}$ are marked by circle, square and triangle, respectively. As the contribution ratio of the mode 1 is increased, the contribution ratio of the mode $2 \mathrm{a}$ is decreased, while the mode $2 \mathrm{~b}$ shows the steady contribution ratio. Furthermore, the sum of the contribution ratio of the modes 1 and $2 \mathrm{a}$ is steady. Thus, the contribution ratio is exchanged between modes 1 and $2 \mathrm{a}$. The relative change of the contribution ratio between the modes 1 and $2 \mathrm{a}$ shows that the modes 1 and 2 a contribute to the chaotic response as the internal resonance, while the steady contribution ratio of the mode $2 b$ shows that the mode $2 b$ contributes to the chaotic response as the superharmonic resonance of second order because the excitation frequency is nearly half the natural frequency of the mode $2 b$.

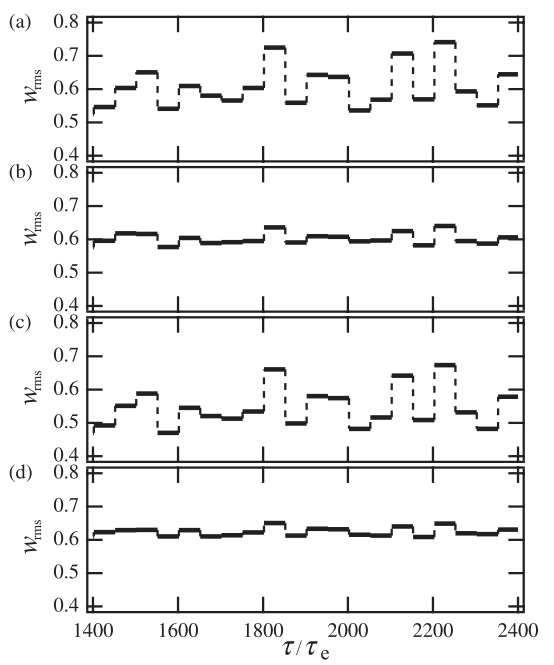

Fig. 11 Time histories of the amplitude $\left(\omega_{\mathrm{ex}}=9.59, p_{d}=4.4\right)$ : (a) measured at $\xi=0.47, \theta=0$; (b) measured at $\xi=0.47, \theta=\pi / 2$; (c) measured at $\xi=0.47$, $\theta=\pi ;$ (d) measured at $\xi=0.47, \theta=3 \pi / 2$.

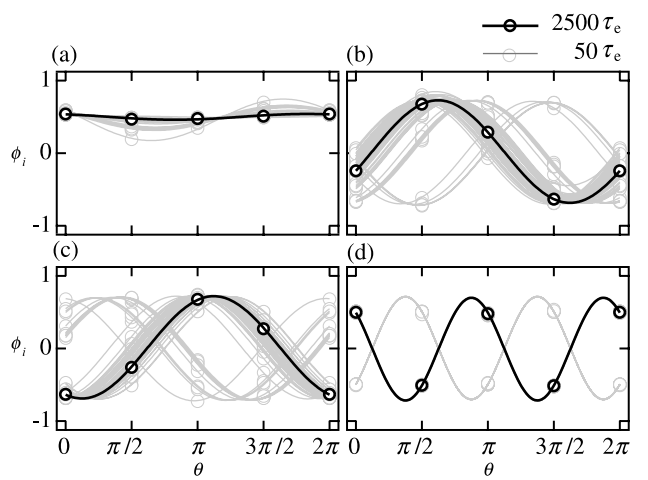

Fig. 12 Modal pattern obtained by Karrhunen-Loève transformation $\left(\omega_{\mathrm{ex}}=9.59\right.$, $p_{d}=4.4$ ): (a) $\mu_{1}$; (b) $\mu_{2}$; (c) $\mu_{3}$; (d) $\mu_{4}$.

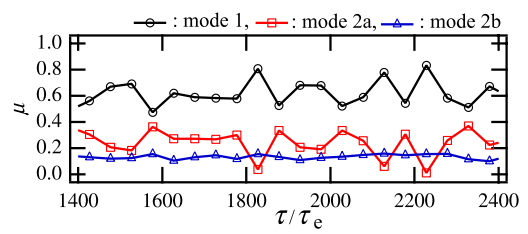

Fig. 13 Time history of the contribution ratio corresponds to the each modal configuration $\left(\omega_{\mathrm{ex}}=9.59, p_{d}=4.4\right)$. 


\section{Conclusions}

Experimental results are presented on chaotic vibrations of a thin circular plate with outer clamped edge and a circular center hole. Main results are summarized as follows.

( 1 ) Second and third modes of vibration with one nodal diameter are generated on different natural frequencies due to an asymmetric curved deformation of the plate. These nodal diameters are perpendicular to each other.

( 2 ) In the restricted range of the principal resonance of the lowest mode, a chaotic response with amplitude modulation is generated. The chaotic response is generated from the one-to-two internal resonance coupled with the lowest and the second modes of vibration.

( 3 ) From the result of principal component analysis, the lowest, the second and the third modes show the predominant contribution ratio of 62,24 and 14 percent, respectively. By changing the calculation of the principal component analysis with the time histories in shorttime intervals, it is found that the nodal patterns of the second and the third modes of vibration are fluctuated along the circumferential direction. Furthermore, the exchange of the contribution ratio is shown between the lowest and the second modes of vibration.

\section{References}

( 1 ) Yamaki, N., Otomo, K., Chiba, M., Nonlinear vibrations of a clamped circular plate with initial deflection and initial edge displacement, part I: theory, Journal of sound and vibration, Vol.79(1), (1981), pp.23-42.

( 2 ) Yamaki, N., Otomo, K., Chiba, M., Nonlinear vibrations of a clamped circular plate with initial deflection and initial edge displacement, part II: experiment, Journal of sound and vibration, Vol.79(1), (1981), pp.43-59.

( 3 ) Arafat, H.N., Nayfeh,A.H. Faris,W., Natural frequencies of heated annular and circular plate, International Journal of Solids and Structures, Vol.41, (2004), pp.3031-3051.

( 4 ) Arafat, H.N., Nayfeh,A.H., Modal interactions in the vibrations of a heated annular plate, International Journal of Non-linear Mechanics, Vol.39, (2004), pp.1671-1685.

( 5 ) Nayfeh,T.A. and Vakakis,A.F., Subharmonic traveling waves in a geometrically non-linear circular plate, International Journal of Non-Linear Mechanics., Vol.29, No.2,(1994), pp.233-245.

( 6 ) Torii, T., Yasuda, K., Toyoda, T., Nonlinear oscillation of a rotating disc excited at a fixed point in space (Combination tones near primary resonance point), Transactions of the Japan Society of Mechanical Engineers, Series C, Vol.60, No.570, (1994), pp.386393.

( 7 ) Wolf, A., Swift, J.B., Swinney, H.L. and Vastano, J.A., Determining Lyapunov exponents from a time series, Physica, D, Vol. 16, (1985), pp. 285-317.

( 8 ) F. Takens, Detecting strange attractors in turbulence, in: D. Rand, L. Young (Eds.), Lecture Notes in Mathematics, Springer, New York, (1981), pp. 366-381.

( 9 ) Nagai, K., Maruyama, S., Murata, T., Yamaguchi, T., Experiments and analysis on chaotic vibrations of a shallow cylindrical shell-panel, Journal of Sound and Vibration, Vol.305, (2007), pp.492-520.

(10) Azeez, M. F. and Vakakis, A. F., Proper orthogonal decomposition (POD) of a class of vibroimpact oscillations, Journal of Sound and Vibration, Vol.240 No.5 (2001), pp.859889.

(11) Nagai, K., Yamaguchi, T., Tanifuji, K., Yoh, H., Experiment on chaotic vibrations of a cantileverd beam deformed by a stretched cable, Transactions of the Japan Society of Mechanical Engineers, Series C, Vol.60, No.569, C(1994), pp.3-9. 